\title{
Trachoma: leading cause of infectious blindness
}

Background and epidemiology:

Trachoma is a chronic keratoconjunctivitis caused by repeated infection with the ocular serovars A, B, Ba and C of Chlamydia trachomatis. ${ }^{1}$ The name, derived from the Greek word for "rough," describes the appearance of the lymphoid follicles apparent with trachomatous inflammation when the upper eyelid is everted and the upper tarsal conjunctiva inspected. A single episode of C. trachomatis ocular infection produces a self-limiting mucopurulent conjunctivitis. Repeated infections lead to conjunctival scarring and distortion of the lid margin, which causes the eyelashes to turn inward (entropion) and repeatedly rub against the cornea (trichiasis). ${ }^{1}$ The catastrophic outcome is corneal opacification and, ultimately, blindness.

Trachoma is second only to cataracts as a cause of blindness and accounts for about $15 \%$ of cases of blindness in the world. In 1997 the World Health Organization (WHO) estimated that 146 million people were actively infected, more than 10 million had trichiasis, and about 6 million were blind from corneal scarring. ${ }^{1,2}$ Active disease is most often seen in children, and women more often than men experience the visual loss that results from chronic, untreated disease, probably because of their greater direct contact with children.

The root of this infectious and preventable disease is poverty. Households affected by trachoma characteristically have young children living with unsafe water and inadequate sanitation. Transmission from eye to eye is by fingers, fomites (e.g., shared towels) and flies. Once endemic in Europe and North America, trachoma has virtually been eliminated from developed countries through improvements in hygiene and sanitation. The burden currently falls on residents of sub-Saharan Africa, northern Africa and parts of the Middle East and Asia. ${ }^{3}$
Clinical management: Laboratory diagnosis is possible using culture, immunofluorescent microscopy, enzyme-linked immunosorbent assay or polymerase chain reaction. However, laboratories are generally unavailable in poor communities where trachoma is endemic, so the diagnosis is usually made clinically. WHO has developed a simplified grading system based on signs and the extent of inflammation, conjunctival thickening and scarring, trichiasis and corneal opacity (Box 1$){ }^{1}$

Trachomatous trichiasis (stage TT) can be corrected surgically through a quick and simple procedure called bilamellar tarsal rotation, which involves division of the upper eyelid tarsal plate with external rotation of the distal margin by use of 3 or 4 sutures. In patients with corneal opacification, the only possible treatment is corneal grafting, although results in cases of trachoma are usually disappointing. Patients with active infection (stages TF and TI) can be given a 6-week course of topical tetracycline therapy or a single oral dose of azithromycin. The utility of antibiotic therapy in affected individuals, however, is questionable given the risk of recurrence. In general, effective management of trachoma must also involve interventions at the community level aimed at improving water quality, access to latrines and personal hygiene, and reducing overcrowding and the density of flies in the environment.

Prevention: Humans are the only reservoir for $C$. trachomatis. Thus, treatment of acute infections along with improved hygiene and sanitation should help to eliminate the disease or at least markedly reduce its frequency. In 1997 WHO founded the Alliance for the Global Elimination of Trachoma, whose aim is to eradicate the disease by the year 2020. . $^{2,3}$ WHO is currently working with member states to implement its SAFE strategy (surgery, antibiotic treatment, facial hygiene and environmental change) in affected communities. The effort has been supported and propelled by Pfizer Inc. through its donation of azithromycin and by the Edna McConnell Clark Foundation through the establishment of the International Trachoma Initiative charity. 3,4 To date, more than 55000 lid surgeries have been performed and more than 6 million doses of azithromycin distributed.

\section{Erica Weir \\ CMAF \\ Shariq Haider}

Division of Infectious Diseases

McMaster University Medical Centre Hamilton, Ont.

David Telio

Faculty of Medicine

University of Toronto

Toronto, Ont.

\section{References}

1. Mabey DC, Solomon AW, Foster A. Trachoma. Lancet 2003;362:223-9.

2. Global elimination of blinding trachoma [WHO EB101.R5]. Geneva: World Health Organization; 1998. Available: www.who.int/pbd/trachoma/ebres98-e htm (accessed 2004 Mar 16).

3. Mecaskey JW, Knirsch CA, Kumaresan JA, Cook JA. The possibility of eliminating blinding trachoma. Lancet Infect Dis 2003;3(11):728-34.

4. Kuper H, Solomon AW, Buchan J, Zondervan M, Foster A, Mabey D. A critical review of the SAFE strategy for the prevention of blinding trachoma. Lancet Infect Dis 2003;3(6):372-81.

\section{Box 1: Simplified grading of trachoma}

TF Trachomatous inflammation, follicular: the presence of 5 or more follicles on the upper tarsal conjunctiva

TI Trachomatous inflammation, intense: pronounced inflammatory thickening of the tarsal conjunctiva that obscures more than $50 \%$ of the normal deep tarsal vessels

TS Trachomatous scarring: the presence of white scars on the tarsal conjunctiva

TT Trachomatous trichiasis: at least 1 eyelash turns in and rubs the eyeball

CO Corneal opacity: easily visible opacity of the cornea that is central and dense enough to obscure at least part of the pupil margin

Source: World Health Organization.' 